\begin{tabular}{|c|l|}
\hline Title & Diversity and mechanisms of al kali tolerance in lactobacilli \\
\hline Author(s) & Sawatari, Yuki; Y okota, A tsushi \\
\hline Citation & $\begin{array}{l}\text { A pplied and Environmental Microbiology, 73(12), 3909-3915 } \\
\text { https://doi.org/10.1128/AEM.02834.06 }\end{array}$ \\
\hline Issue Date & 2007-06 \\
\hline Doc URL & http://hdl.handle.net/2115/28128 \\
\hline Rights & Copyright $\odot$ A merican Society for Microbiology. \\
\hline Type & article \\
\hline File Information & AEM7312.pdf \\
\hline
\end{tabular}

Instructions for use 


\title{
Diversity and Mechanisms of Alkali Tolerance in Lactobacilli ${ }^{\nabla}$
}

\author{
Yuki Sawatari and Atsushi Yokota* \\ Laboratory of Microbial Physiology, Research Faculty of Agriculture, Hokkaido University, Kita 9 Nishi 9 , \\ Kita-ku, Sapporo, Hokkaido 060-8589, Japan
}

Received 6 December 2006/Accepted 8 April 2007

\begin{abstract}
We determined the maximum $\mathrm{pH}$ that allows growth (pHmax) for 34 strains of lactobacilli. High alkali tolerance was exhibited by strains of Lactobacillus casei, L. paracasei subsp. tolerans, L. paracasei subsp. paracase $i, L$. curvatus, $L$. pentosus, and $L$. plantarum that originated from plant material, with pHmax values between 8.5 and 8.9. Among these, $L$. casei NRIC 1917 and $L$. paracasei subsp. tolerans NRIC 1940 showed the highest pHmax, at 8.9. Digestive tract isolates of $L$. gasseri, $L$. johnsonii, $L$. reuteri, L. salivarius subsp. salicinius, and $L$. salivarius subsp. salivarius exhibited moderate alkali tolerance, with pHmax values between 8.1 and 8.5 . Dairy isolates of $L$. delbrueckii subsp. bulgaricus, $L$. delbrueckii subsp. lactis, and $L$. helveticus exhibited no alkali tolerance, with pHmax values between 6.7 and 7.1. Measurement of the internal $\mathrm{pH}$ of representative strains revealed the formation of transmembrane proton gradients $(\Delta \mathrm{pH})$ in a reversed direction (i.e., acidic interior) at alkaline external-pH ranges, regardless of their degrees of alkali tolerance. Thus, the reversed $\Delta \mathrm{pH}$ did not determine alkali tolerance diversity. However, the $\Delta \mathrm{pH}$ contributed to alkali tolerance, as the $\mathrm{pHmax}$ values of several strains decreased with the addition of nigericin, which dissipates $\Delta \mathrm{pH}$. Although neutral external-pH values resulted in the highest glycolysis activity in the presence of nigericin regardless of alkali tolerance, substantial glucose utilization was still detected in the alkali-tolerant strains, even in a pH range of between 8.0 and 8.5, at which the remaining strains lost most activity. Therefore, the alkali tolerance of glycolysis reactions contributes greatly to the determination of alkali tolerance diversity.
\end{abstract}

Lactic acid bacteria (LAB) produce lactic acid as a main product of sugar fermentation. Many studies have examined the response of LAB to acid stress, including the mechanisms underlying acid tolerance $(3,4,28)$. In contrast, very few studies have examined the stress response of LAB to alkaline environments. Several alkali-tolerant/alkaliphilic LAB, which are capable of growing at $\mathrm{pH}$ values of $>9.5$ as well as producing large amounts of lactic acid from glucose, have been reported to date: Enterococcus hirae (formerly Streptococcus faecalis) (8), Pediococcus urinaeequi (23), Alkalibacterium olivapovliticus (12, 24), Marinilactibacillus psychrotolerans (12), M. piezotolerans (27), Halolactibacillus halophilus, and H. miurensis (13).

The mechanism underlying the alkali tolerance of LAB has been examined in E. hirae ATCC $9790^{\mathrm{T}}$. Biochemical experiments and mutant analyses suggest that $E$. hirae ATCC $9790^{\mathrm{T}}$ maintains an internal $\mathrm{pH}$ lower than the external $\mathrm{pH}$ by the operation of an ATP-driven $\mathrm{K}^{+} / \mathrm{H}^{+}$antiporter during growth at an external $\mathrm{pH}$ of around 9.5 (14-17). However, E. hirae ATCC $9790^{\mathrm{T}}$ has no such system to maintain a near-neutral internal $\mathrm{pH}$ in an alkaline medium (22). Thus, the contribution of internal-pH maintenance to alkali tolerance in this bacterium is controversial. Adaptation to alkaline stress has been reported for E. faecalis ATCC $19433^{\mathrm{T}}$, in which the induction of two stress proteins, DnaK and GroEL, was detected (9).

Lactobacilli, although generally not considered alkali tolerant, have been used as a starter for the fermentation of alkaline

\footnotetext{
* Corresponding author. Mailing address: Laboratory of Microbial Physiology, Division of Applied Bioscience, Research Faculty of Agriculture, Hokkaido University, Kita 9 Nishi 9, Kita-ku, Sapporo, Hokkaido 060-8589, Japan. Phone: 81-11-706-2501. Fax: 81-11-706-4961. E-mail: yokota@chem.agr.hokudai.ac.jp.

${ }^{\nabla}$ Published ahead of print on 20 April 2007.
}

materials to prepare fermented foods. For example, Lactobacillus pentosus CECT 5138 has been studied as a starter to ferment lye-treated green olives $(\mathrm{pH},>9.0)$ for the preparation of Spanish-style green olives (25). In our previous study (26), in which noodle dough with a $\mathrm{pH}$ of around 8.5 was fermented, $L$. plantarum NRIC 0380 was screened as a starter strain for fermented instant Chinese noodles.

Because there has been little examination of alkali tolerance and sensitivity in Lactobacillus strains, we investigated the effects of alkaline stress on the growth, physiology, and metabolism of lactobacilli from various sources for more effective use of this group of bacteria in food industries.

\section{MATERIALS AND METHODS}

Bacterial strains and chemicals. The bacterial strains used (Table 1) were obtained from the Japan Collection of Microorganisms (JCM, Wako, Japan), the Culture Collection Center, Tokyo University of Agriculture (NRIC, Tokyo, Japan); the Laboratory of Applied Microbiology, Research Faculty of Agriculture, Hokkaido University (AHU, Sapporo, Japan); and the Wageningen Cente for Food Sciences (Wageningen, The Netherlands). [(2-Hydroxy-1,1-bis(hydroxymethyl)ethyl)amino]-1-propanesulfonic acid; $N$-[Tris(hydroxymethyl)methyl]-3 aminopropanesulfonic acid (TAPS); $N$-Tris(hydroxymethyl)methyl-4-aminobutanesulfonic acid (TABS); 2-(cyclohexylamino)ethanesulfonic acid (CHES); and nigericin were obtained from Sigma-Aldrich (St. Louis, MO). 2-(Morpholino)ethanesulfonic acid monohydrate (MES) and 2-[4-(2-hydroxyethyl)-1-piperazinyl]ethanesulfonic acid (HEPES) were obtained from Dojindo Laboratories (Mashikimachi, Kumamoto, Japan). 3-( $N$-morpholino)propanesulfonic acid (MOPS) was purchased from Nacalai Tesque (Kyoto, Japan). The 5 (and 6)carboxyfluorescein diacetate succinimidyl ester (cFDASE) was obtained from Molecular Probes (Eugene, OR). Other chemicals used were of the highes quality available commercially.

Determination of maximum alkaline pH allowing growth of lactobacilli. For the preparation of seed cultures, frozen stocks of Lactobacillus strains and $E$. hirae JCM $8729^{\mathrm{T}}$ kept under $-80^{\circ} \mathrm{C}$ were transferred to $3 \mathrm{ml}$ of half-strength MRS (1/2 MRS) medium (6) and incubated overnight at $30^{\circ} \mathrm{C}$ or $37^{\circ} \mathrm{C}$, as indicated in the legend to Table 1 . All cultures were grown in screw-cap test tubes tightly sealed with rubber septums. No other anaerobic treatment was 
TABLE 1. Determination of the maximum $\mathrm{pH}$ that allows the growth of various Lactobacillus strains and E. hirae JCM $8729^{\mathrm{Ta}}$

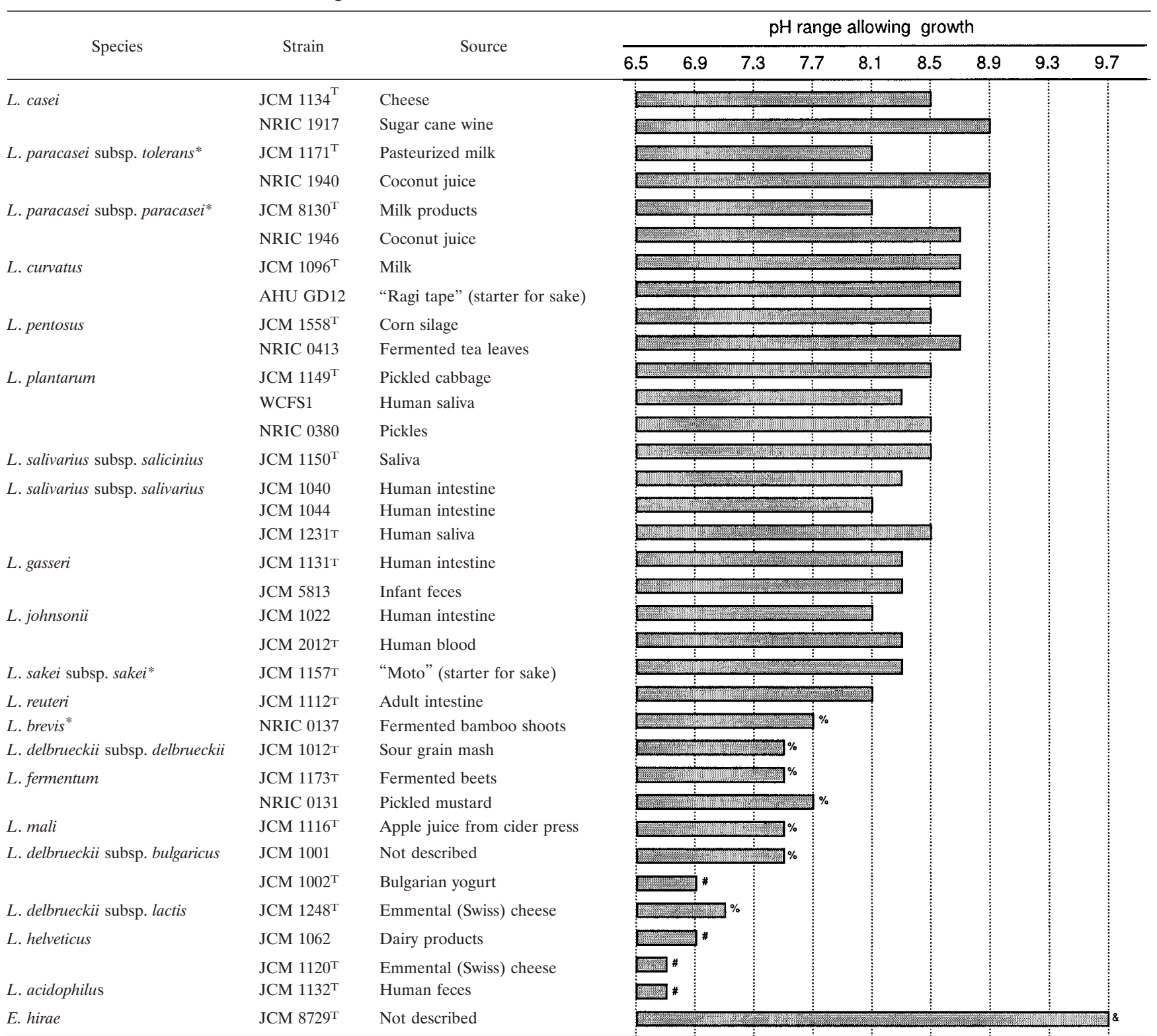

${ }^{a}$ Gray bars indicate the $\mathrm{pH}$ range allowing growth for each strain. Cultures were grown in buffered $1 / 2 \mathrm{MRS}$ media at $37^{\circ} \mathrm{C}$, except for species denoted with asterisks, which were cultured at $30^{\circ} \mathrm{C}$. Buffers used in the buffered $1 / 2$ MRS medium included MOPS (\#), HEPES (\%), and CHES (\&); TAPS buffer was used for the rest of the cultures. The data are derived from at least two independent experiments that gave similar results. When different pHmax values were obtained for a given strain, the lower value was adopted.

applied. The 1/2 MRS medium contained $10 \mathrm{~g} /$ liter glucose, $5 \mathrm{~g}$ /liter Bacto Proteose Peptone No. 3 (Becton Dickinson, Franklin Lakes, NJ), 5 g/liter LabLemco powder (Oxoid, Basingstoke, Hampshire, UK), $2.5 \mathrm{~g} /$ liter Bacto yeast extract (Becton Dickinson), $0.5 \mathrm{~g} /$ liter Tween 80, $1 \mathrm{~g}$ /liter $\mathrm{K}_{2} \mathrm{HPO}_{4}, 2.5 \mathrm{~g} /$ liter $\mathrm{NaCH}_{3} \mathrm{CO}_{2} \cdot 3 \mathrm{H}_{2} \mathrm{O}, 1 \mathrm{~g} /$ /iter triammonium citrate, $0.1 \mathrm{~g} /$ liter $\mathrm{MgSO}_{4} \cdot 7 \mathrm{H}_{2} \mathrm{O}$, and $0.025 \mathrm{~g} /$ liter $\mathrm{MnSO}_{4} \cdot 4$ to $5 \mathrm{H}_{2} \mathrm{O}$; the $\mathrm{pH}$ was adjusted to 6.5 , using $\mathrm{HCl}$. The seed culture was inoculated into $3 \mathrm{ml}$ of buffered $1 / 2 \mathrm{MRS}$ medium at an optical density at $660 \mathrm{~nm}\left(\mathrm{OD}_{660}\right)$ of around 0.03 . The buffered 1/2 MRS medium was prepared by supplementing $1 / 2$ MRS medium with an appropriate buffer at 100 $\mathrm{mM}$ to prevent acidification of the culture medium, using the following buffers: MOPS (pH 6.5 to 7.9), HEPES (pH 7.1 to 8.1), TAPS (pH 8.1 to 9.1), and CHES (pH 8.7 to 9.7$)$. The $\mathrm{pH}$ of these media was adjusted using $\mathrm{NaOH}$ or $\mathrm{HCl}$ Growth experiments using the lactobacilli strains were conducted at a $\mathrm{pH}$ range of 6.5 to 9.1; experiments using E. hirae $\mathrm{JCM} 8729^{\mathrm{T}}$ were conducted at a pH range of 6.5 to 9.7. Incubation was conducted at the same temperatures and same anaerobic conditions as used for the seed cultures for up to $24 \mathrm{~h}$, and growth was monitored by the $\mathrm{OD}_{660}$, using a spectrophotometer (Spectronic $20 \mathrm{D}+$; Milton Roy Company). Strains that reached an $\mathrm{OD}_{660}$ of $>0.15$ within $24 \mathrm{~h}$ of being cultured were considered to exhibit positive growth. At this point, acidification of the media appeared to be negligible (around $0.05 \mathrm{pH}$ unit). Additionally, specific growth rates $\left(\mu\right.$; expressed in $\mathrm{h}^{-1}$ ) were calculated during the exponential growth phase, using the following equation: $\mu=\left(\ln x_{t 2}-\ln x_{t 1}\right) /(t 2-t 1)$, where $x_{t 2}$ and $x_{t 1}$ are the $\mathrm{OD}_{660}$ at times $t 2$ and $t 1$, respectively. Normally, calculation was conducted at the early exponential phase $\left(\mathrm{OD}_{660}, \sim 0.3\right)$, at which point acidification of the medium (around $0.15 \mathrm{pH}$ unit) did not seem to affect $\mu$ values significantly.

Intracellar $\mathbf{p H}$ measurement. The internal $\mathrm{pH}$ of five representative strains of lactobacilli (see Fig. 2) and E. hirae JCM $8729^{\mathrm{T}}$ was measured at external-pH 


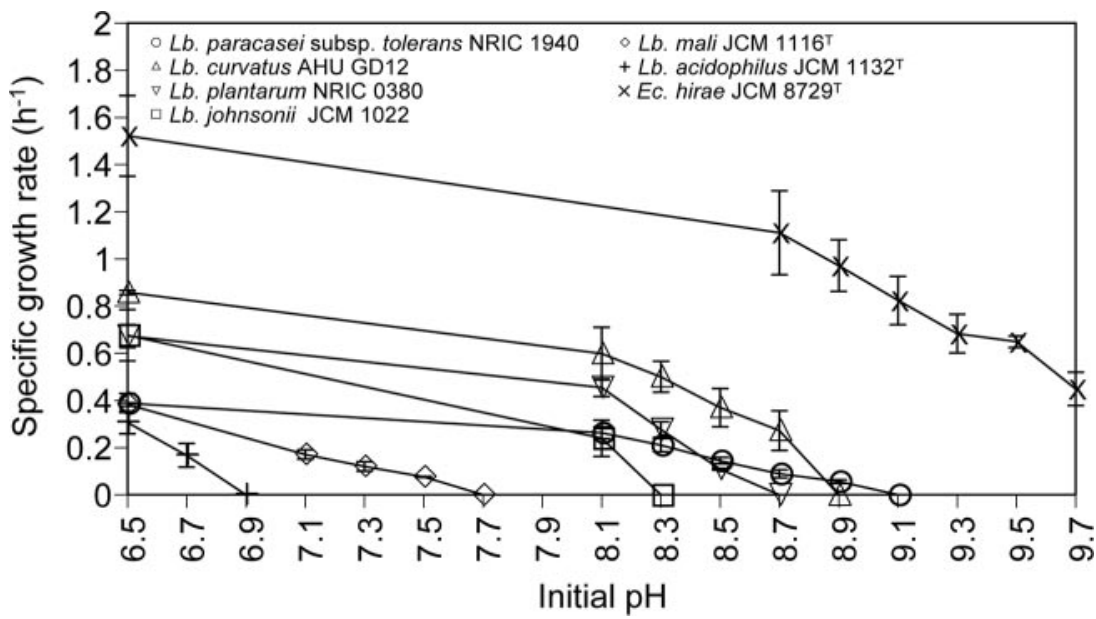

FIG. 1. Specific growth rates of representative Lactobacillus $\left(L b\right.$. ) strains and Enterococcus $\left(\right.$ Ec.) hirae JCM $8729^{\mathrm{T}}$. The strains were cultured in buffered 1/2 MRS media. Each point represents the mean \pm the standard deviation of the results of two independent experiments.

values of 6.5 to 9.0, using the pH-sensitive fluorescence probe 5 (and 6-)carboxyfluorescein succinimidyl ester (cFSE), based on the method originally described by Breeuwer et al. (2), with some modification. The fluorescence intensity of this probe is increased at alkaline $\mathrm{pH}$ and reduced at acidic $\mathrm{pH}$. Seed culture was inoculated into an appropriate volume of $1 / 2$ MRS medium in a screw-cap bottle at an $\mathrm{OD}_{660}$ of about 0.05 . The culture was conducted to an $\mathrm{OD}_{660}$ of around 0.6 (i.e., exponential growth phase) under the same conditions as described in the preceding section. The cultured cells were harvested by centrifugation at $8,000 \times g$ for 5 min at $4^{\circ} \mathrm{C}$ and washed twice with buffer $\mathrm{A}$ (150 $\mathrm{mM}$ potassium phosphate buffer with $1 \mathrm{mM} \mathrm{MgSO}_{4} \cdot 7 \mathrm{H}_{2} \mathrm{O}[\mathrm{pH}$ 6.5]). The cells were resuspended in buffer A containing $4 \mu \mathrm{M}$ cFDASE, a precursor molecule of cFSE, at an $\mathrm{OD}_{660}$ of around 0.5 and incubated for $30 \mathrm{~min}$ at the temperatures described in the footnote to Table 1. During this incubation, the membranepermeating cFDASE was cleaved by intracellular esterases, and the resultant cFSE molecules were conjugated to the aliphatic amines of intracellular proteins. After being centrifugated and washed with buffer A, the cells were resuspended in the same volume of buffer A. The nonconjugated probe was eliminated by the addition of glucose at a final concentration of $10 \mathrm{mM}$ and subsequent incubation for $1 \mathrm{~h}$ at the temperatures described in the footnote to Table 1. After being centrifugated and washed with buffer A, the cells were resuspended in buffer A, and their energy was depleted through incubation for $1 \mathrm{~h}$ at the temperatures described in the footnote to Table 1 . After being centrifugated and washed with buffer $\mathrm{A}$, the cells were resuspended in buffer $\mathrm{A}$ at an $\mathrm{OD}_{660}$ of around 6 . A $100-\mu \mathrm{l}$ aliquot of this cell suspension was diluted in $1.9 \mathrm{ml}$ of buffer B (buffer A supplemented with $100 \mathrm{mM}$ MOPS or TAPS [pH 6.5]) in a cuvette, and the cuvette was placed in a stirred and heated (same culture temperature) cuvette holder in an LS50B fluorimeter (PerkinElmer Life and Analytical Sciences, Wellesley, MA). The internal $\mathrm{pH}$ was determined by measuring the fluorescence intensities of the cell suspension with excitation and emission wavelengths of 490 and $520 \mathrm{~nm}$, respectively (slit widths of $2.5 \mathrm{~nm}$ ). In the experiments, while the fluorescence intensity was being monitored, the cells were energized with $10 \mathrm{mM}$ glucose at $\mathrm{pH} 6.5$ and incubated until the intensity stabilized. After that, the $\mathrm{pH}$ of the mixture was increased to the desired values between 7.0 and 9.0, using $\mathrm{NaOH}$ or $\mathrm{KOH}$ (see Fig. 2), and the mixture was incubated for 30 min more. Subsequently, the cells were treated with nigericin, which dissipates transmembrane proton gradient $(\Delta \mathrm{pH})$, at a final concentration of $2 \mu \mathrm{M}$, and the fluorescence intensity was measured for calibration at appropriate external-pH values adjusted with the corresponding alkali $(\mathrm{NaOH}$ or $\mathrm{KOH})$ and $\mathrm{HCl}$.

Growth in the presence of nigericin. To evaluate the effect of $\Delta \mathrm{pH}$ on alkali tolerance, growth experiments using four strains of lactobacilli (see Fig. 3A) and E. hirae JCM $8729^{\mathrm{T}}$ were conducted in the presence or absence of nigericin at $\mathrm{pH}$ values between 6.5 and 9.0. Both seed cultures and main cultures were grown as described under "Determination of the maximum alkaline $\mathrm{pH}$ allowing the growth of lactobacilli," with the following modifications. Potassium-enriched $1 / 2$ MRS (K-1/2 MRS) medium was used for the seed cultures and as the basal medium for the main cultures, and the main cultures were grown in the presence or absence of nigericin. Potassium was added to avoid possible growth inhibition caused by nigericin, as was previously observed in E. hirae ATCC $9790^{\mathrm{T}}(\mathrm{JCM}$
$8729^{\mathrm{T}}$ ) because of the loss of $\mathrm{K}^{+}$from the cells (10). K-1/2 MRS medium was prepared by modifying $1 / 2 \mathrm{MRS}$ medium. The concentration of $\mathrm{K}_{2} \mathrm{HPO}_{4}$ was increased to $10 \mathrm{~g} /$ liter, and $\mathrm{NaCH}_{3} \mathrm{CO}_{2} \cdot 3 \mathrm{H}_{2} \mathrm{O}$ was replaced with $1.8 \mathrm{~g} /$ liter of potassium acetate. The following buffers at $100 \mathrm{mM}$ were added to the $\mathrm{K}-1 / 2$ MRS medium for the main cultures, and the $\mathrm{pH}$ was adjusted to the desired values using $\mathrm{KOH}$ (buffered K-1/2 MRS medium): MES ( $\mathrm{pH} 6.5$ ), MOPS (pH 7.0 to 7.5 ), and TAPS ( $\mathrm{pH} 8.0$ to 9.0). Nigericin in ethanol solution was added at a final concentration of $7 \mu \mathrm{M}$. Control cultures received $0.1 \%$ (vol/vol) ethanol. Specific growth rates were calculated during the exponential growth phase as described above. Acidification of the culture medium during the calculation appeared to be at negligible levels owing to the added buffer system.

Measurement of glycolysis activity in the presence of nigericin. To evaluate the $\mathrm{pH}$ dependency of lactobacilli glycolysis activity, the activities of the four strains used in the preceding section together with that of E. hirae JCM $8729^{\mathrm{T}}$ were measured in the presence of nigericin at various pHs between 4.0 and 9.5. The cells were cultured until the exponential phase $\left(\mathrm{OD}_{660}=0.6\right)$ in $1 / 2 \mathrm{MRS}$ medium, harvested, and washed with buffer A, as described under "Intracellar pH measurement." The washed cell pellet was resuspended in the same buffer and incubated for $1 \mathrm{~h}$ at the same temperatures in which the cells were cultured to deplete their metabolic energy. The cells were washed once with buffer A and resuspended in the same buffer at an $\mathrm{OD}_{660}$ of about 80 . The basal composition of the reaction mixture for the glycolysis reaction contained $150 \mathrm{mM}$ potassium phosphate buffer, $1 \mathrm{mM} \mathrm{MgSO} 4 \cdot 7 \mathrm{H}_{2} \mathrm{O}$, and one of the following buffers at 100 $\mathrm{mM}$ with the $\mathrm{pH}$ adjusted to the desired values, using $\mathrm{KOH}$ : MES ( $\mathrm{pH} 4.0$ to 7.0), MOPS ( $\mathrm{pH} 7.5$ ), TAPS ( $\mathrm{pH} 8.0$ to 9.0), or TABS ( $\mathrm{pH} 9.5$ ). Subsequently, the energy-depleted cells were added at an $\mathrm{OD}_{660}$ of about 4 , and then nigericin in ethanol solution was added at a final concentration of $7 \mu \mathrm{M}$. The control series received $0.1 \%(\mathrm{vol} / \mathrm{vol})$ ethanol. The reaction was started by the addition of glucose at a final concentration of $10 \mathrm{mM}$. The mixtures were incubated at the temperature in which they were cultured, for appropriate time periods (see Fig. 3). After being incubated, the mixtures were centrifuged at $18,120 \times g$ for $5 \mathrm{~min}$ at $4^{\circ} \mathrm{C}$, and the supernatant was filtered through a $0.2-\mu \mathrm{m}$-pore-size membrane. The glucose concentrations of the filtered supernatants were measured, using Glucose C2 (Wako Pure Chemical Industries, Osaka, Japan).

\section{RESULTS}

Maximum pH allowing the growth of lactobacilli. We determined the maximum $\mathrm{pH}$ that allows growth (pHmax) for 34 strains of lactobacilli from various sources. We used $E$. hirae JCM $8729^{\mathrm{T}}$, a well-known alkali-tolerant strain (8), as a reference. As expected, E. hirae JCM $8729^{\mathrm{T}}$ showed growth up to a $\mathrm{pH}$ of 9.7 (Table 1). Relatively high-alkali-tolerant strains in the lactobacilli included the plant isolates of L. casei, L. paracasei subsp. tolerans, L. paracasei subsp. paracasei, L. curvatus, 

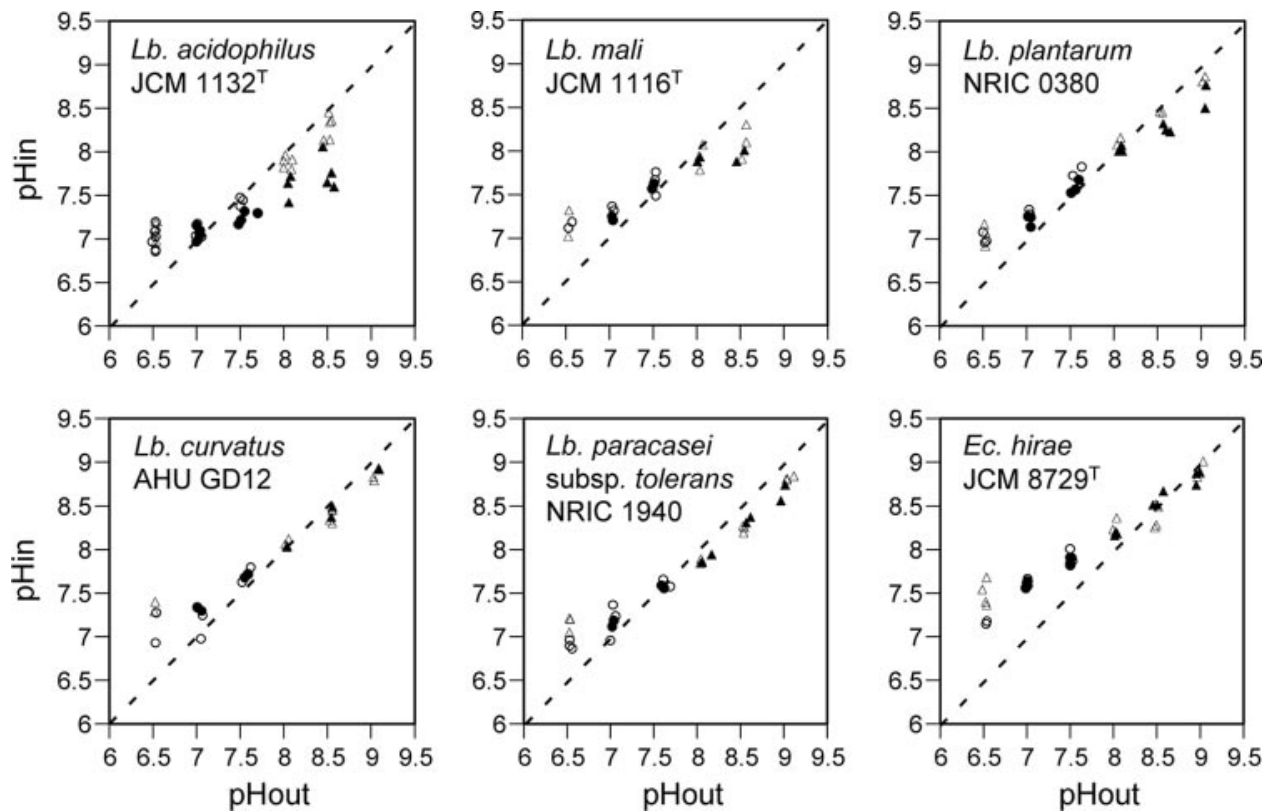

FIG. 2. Internal-pH measurements at various external pHs. MOPS buffer $(\bigcirc)$ or TAPS buffer $(\triangle)$ was used in buffer B (see Materials and Methods). The $\mathrm{pH}$ of buffer $\mathrm{B}$ was adjusted using either $\mathrm{KOH}$ (open symbols) or $\mathrm{NaOH}$ (filled symbols). The dashed lines represent the intracellular $\mathrm{pH}$ (pHin) which is equal to the extracellular $\mathrm{pH}$ (pHout). Lb., Lactobacillus; Ec., Enterococcus.

L. pentosus, and L. plantarum, with pHmax values between 8.5 and 8.9. Of these, L. casei NRIC 1917 and L. paracasei subsp. tolerans NRIC 1940 exhibited the highest alkali tolerance, with a pHmax of 8.9. Within the same species of lactobacilli, i.e., $L$. casei, L. paracasei subsp. tolerans, and L. paracasei subsp. paracasei, isolates from plant materials showed higher alkali tolerance than those from other sources. In contrast, strains of $L$. brevis, L. delbrueckii subsp. delbrueckii, L. fermentum, and L. mali that were isolated from plant materials showed lower pHmax values of between 7.5 and 7.7. Strains of L. gasseri, $L$. johnsonii, L. reuteri, L. salivarius subsp. salicinius, and L. salivarius subsp. salivarius, which were isolated from digestive tracts, exhibited moderate alkali tolerance, with pHmax values between 8.1 and 8.5. The dairy isolates of $L$. delbrueckii subsp. bulgaricus, L. delbrueckii subsp. lactis, and L. helveticus showed no alkali tolerance, with pHmax values between 6.7 and 7.1. The least-alkali-tolerant strains among those tested were $L$. helveticus JCM $1120^{\mathrm{T}}$ and L. acidophilus JCM $1132^{\mathrm{T}}$, which had pHmax values as low as 6.7.

Among six representative strains of lactobacilli and E. hirae $\mathrm{JCM} 8729^{\mathrm{T}}$, E. hirae $\mathrm{JCM} 8729^{\mathrm{T}}$ showed the highest $\mu$ as a function of the initial $\mathrm{pH}$ of the culture medium under the tested $\mathrm{pH}$ range of between 6.5 and 9.7 (Fig. 1). However, in all strains, $\mu$ decreased with the increasing initial $\mathrm{pH}$ of the medium.

Intracellular pH measurements. Next, we clarified the mechanism(s) determining the diversity of alkali tolerance. Previous studies suggested that antiporters acidify cytoplasmic $\mathrm{pH}$ under alkaline conditions in several alkali-tolerant species, including E. hirae ATCC $9790^{\mathrm{T}}$ (15-17), and some alkaliphiles $(20,21)$. To verify the contribution of this mechanism in alkali tolerance, internal-pH measurements were performed, using five strains of lactobacilli with different $\mathrm{pHmax}$ values and $E$. hirae $\mathrm{JCM} 8729^{\mathrm{T}}$ under a range of external-pH values from 6.5 to 9.0 (Fig. 2). An ATP-driven $\mathrm{K}^{+} / \mathrm{H}^{+}$antiporter has been suggested for $E$. hirae ATCC 9790 ${ }^{\mathrm{T}}$ (JCM 8729 ${ }^{\mathrm{T}}$ ) (15-17), whereas $\mathrm{Na}^{+} / \mathrm{H}^{+}$antiporter activity has been implied for the alkaliphilic bacteria Bacillus firmus RAB (20) and Exiguobacterium aurantiacum NCIB 11798 (21). Thus, both $\mathrm{NaOH}$ and $\mathrm{KOH}$ were used to adjust the assay mixtures for $\mathrm{pH}$ measurements. At an external $\mathrm{pH}$ of 6.5 , an $\Delta \mathrm{pH}$ (i.e., an alkaline interior) was generated in all tested strains by energization with glucose. When the external $\mathrm{pH}$ was increased using $\mathrm{KOH}$, the internal $\mathrm{pH}$ also rose, but at a slower rate, thereby decreasing the $\Delta \mathrm{pH}$. Above a specific external $\mathrm{pH}, \Delta \mathrm{pH}$ was reversed (i.e., the interior became acidic), and under highly alkaline external $\mathrm{pH}$, the internal $\mathrm{pH}$ increased while maintaining the magnitude of the reversed $\Delta \mathrm{pH}$ at about 0.5 in all tested strains, including E. hirae JCM $8729^{\mathrm{T}}$. We did not observe the remarkable cytoplasmic $\mathrm{pH}$ regulation previously reported at alkaline external pH in E. hirae ATCC $9790^{\mathrm{T}}$ (14). The formation of the reversed $\Delta \mathrm{pH}$ was observed not only in the highly alkali-tolerant strains E. hirae JCM $8729^{\mathrm{T}}$ and L. paracasei subsp. tolerans NRIC 1940, but also in the least-alkali-tolerant strain, L. acidophilus JCM $1132^{\mathrm{T}}$ (Fig. 2). Similar results were obtained when $\mathrm{NaOH}$, rather than $\mathrm{KOH}$, was used to adjust the $\mathrm{pH}$, except that a significantly larger reversed $\Delta \mathrm{pH}$ was observed in L. acidophilus JCM $1132^{\mathrm{T}}$ than that observed when $\mathrm{KOH}$ was used for $\mathrm{pH}$ adjustment. Internal-pH regulation does not influence the diversity of lactobacilli alkali tolerance.

Growth in the presence of nigericin. The reversed $\Delta \mathrm{pH}$ observed in the preceding experiments was expected to contribute to growth in alkaline conditions. To verify this, we examined growth in the presence or absence of nigericin, which dissipates $\Delta \mathrm{pH}$, using four strains of lactobacilli and $E$. hirae JCM $8729^{\mathrm{T}}$ in buffered K-1/2 MRS. We observed dramatic decreases in the $\mu$ values of all the Lactobacillus strains and $E$. 
(A)
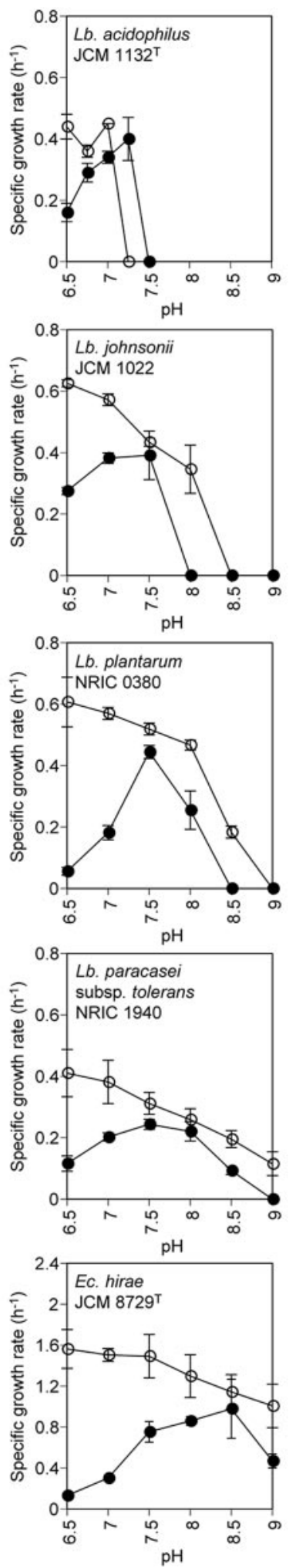

(B)
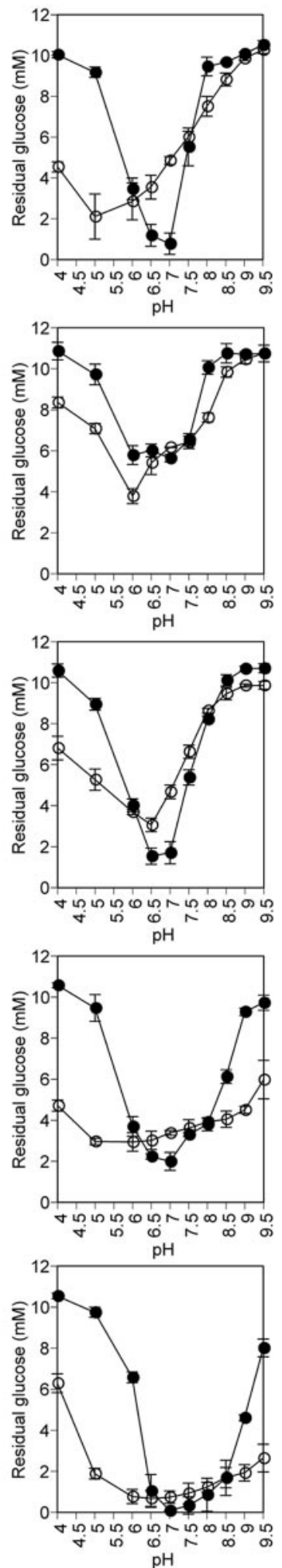

FIG. 3. (A) Specific growth rates and (B) glycolysis activities of representative Lactobacillus (Lb.) strains and Enterococcus (Ec.) hirae JCM $8729^{\mathrm{T}}$. The experiments were carried out in the presence (filled symbols) or absence (open symbols) of nigericin. Glycolysis reactions were conducted for the following time periods: L. acidophilus JCM $1132^{\mathrm{T}}$ and $L$. paracasei subsp. tolerans NRIC 1940, $2 \mathrm{~h} ; L$. johnsonii

hirae $\mathrm{JCM} 8729^{\mathrm{T}}$ in the presence of nigericin under a range of acidic $\mathrm{pH}$ from 6.5 to 7.0 (Fig. 3A). This was expected from previous studies showing that maintenance of intracellular $\mathrm{pH}$ by $\mathrm{H}^{+}$-ATPase is important for growth and viability in acidic conditions $(1,18,29)$. Under a range of alkaline $\mathrm{pH}$, the $\mu$ values of $L$. johnsonii JCM 1022, L. plantarum NRIC 0380, and L. paracasei subsp. tolerans NRIC 1940 were decreased in the presence of nigericin and the pHmax of each strain was decreased by 0.5 . Thus, the reversed $\Delta \mathrm{pH}$ formed in alkaline conditions contributes to the alkali tolerance of the tested Lactobacillus strains. Furthermore, we observed that the $\mathrm{pHmax}$ of a given strain in the presence of nigericin was positively dependent on the pHmax in the absence of nigericin (Fig. 3A), suggesting that the degree of resistance of the cellular metabolism, including catabolism and anabolism to an alkaline environment, may determine the alkali tolerance of a given strain.

Glycolysis activity in the presence of nigericin. To evaluate the preceding hypothesis, we measured the glycolysis activity of the strains in the presence or absence of nigericin at various external $\mathrm{pH}$ values. As expected, we observed dramatic decreases in glycolysis activity at a range of acidic $\mathrm{pH}$ values for all strains tested (Fig. 3B). The glycolysis activities of the highly alkali-tolerant strains NRIC 1940 and $E$. hirae JCM $8729^{\mathrm{T}}$ were also greatly decreased at a range of alkaline $\mathrm{pH}$ above 9.0 in the presence of nigericin (Fig. 3B). However, the optimum $\mathrm{pH}$ for glycolysis activity in the presence of nigericin of all strains tested was neutral, regardless of the strain's alkali tolerance. Interestingly, substantial glucose utilization in the highly alkalitolerant strains NRIC 1940 and E. hirae JCM 8729 ${ }^{\mathrm{T}}$ was still detected up to a $\mathrm{pH}$ range of between 8.0 and 8.5 in the presence of nigericin, whereas the remaining strains lost most glycolysis activity. Therefore, the diversity of alkali tolerance is strongly dependent on the alkali tolerance of cellular metabolism, especially glycolysis reactions involved in the generation of energy.

\section{DISCUSSION}

We determined the pHmax of many lactobacilli for the first time. The pHmax ranged between 6.7 and 8.9, demonstrating the diversity of alkali tolerance in lactobacilli (Table 1). Strains of L. casei, L. paracasei subsp. tolerans, L. paracasei subsp. paracasei, L. curvatus, L. pentosus, and L. plantarum originating from plant material exhibited high alkali tolerance, with pHmax values of $>8.5$. It is not clear if there is a correlation between the plant environment and the strain's alkali tolerance. Most of these strains belong to two phylogenetic groups of lactobacilli: the L. casei group (strains tested, L. casei, L. paracasei subsp. paracasei, and L. paracasei subsp.tolerans) and the $L$. plantarum group (strains tested, L. plantarum and $L$. pentosus) (5). Therefore, we speculated that strains belonging to these groups may have a tendency for alkali tolerance from an evolutionary viewpoint. The next-highest alkali-tolerant

JCM 1022, 1.5 h; L. plantarum NRIC 0380 and E. hirae JCM $8729^{\mathrm{T}}$, $1 \mathrm{~h}$. Each point represents the mean \pm the standard deviation of the results of two independent experiments. 
group contained strains of $L$. gasseri, L. johnsonii, L. reuteri, $L$. salivarius subsp. salicinius, and $L$. salivarius subsp. salivarius isolated from digestive tracts; these ranged in pHmax from 8.1 to 8.5 . The small intestine, which is colonized by various intestinal bacteria, including lactobacilli and E. hirae, has a weakly alkaline environment of around $\mathrm{pH} 7.5$ (7). Thus, we assumed that Lactobacillus strains originating in human intestine would exhibit relatively high $\mathrm{pHmax}$ as a result of adaptation to the alkaline environment of the small intestine. L. acidophilus JCM $1132^{\mathrm{T}}$ seems an exceptional strain, with the pHmax as low as 6.7. However, as discussed later in the Discussion section, this strain possibly has certain system(s) to create significantly larger reversed $\Delta \mathrm{pH}$ under alkaline conditions than any other strain tested (Fig. 2), suggesting survival under an alkaline environment (without growth). We found extremely low alkali tolerance in the dairy strains of $L$. delbrueckii subsp. bulgaricus, L. delbrueckii subsp. lactis, and L. helveticus, with pHmax values between 6.7 and 7.1. Dairy starter fermentations generally proceed under severe acidic conditions of around $\mathrm{pH} 4$. Thus, dairy strains may have little chance of encountering an alkaline environment and, therefore, have not evolved high tolerance.

We found that the diversity of alkali tolerance depended strongly on the alkali tolerance of the cellular metabolism, including catabolism and anabolism, as observed in terms of growth (Fig. 3A), especially on the glycolysis reactions of a given strain (Fig. 3B). In fact, the alkali-tolerant reference strain, E. hirae JCM $8729^{\mathrm{T}}$, exhibited the optimum $\mathrm{pH}$ for growth of 8.5 in the presence of nigericin, which is significantly higher than those of the other tested strains, which showed the $\mathrm{pH}$ optima to be around 7.5 (Fig. 3A). The $\mathrm{pH} /$ glycolysis activity profile of $E$. hirae JCM $8729^{\mathrm{T}}$ also shifted to the alkaline side compared to those of the other tested strains in the presence of nigericin (Fig. 3B). A dramatic decrease in glycolysis activity, which was not observed in the other strains, occurred at pH 6.0 (Fig. 3B). This shift in the $\mathrm{pH} /$ glycolysis activity profile was also observed to a lesser extent in L. paracasei subsp. tolerans NRIC 1940, which showed the highest alkali tolerance of the tested lactobacilli (Fig. 3B). Nevertheless, the optimal $\mathrm{pH}$ for glycolysis activity was neutral in all strains, regardless of their alkali tolerance (Fig. 3B), which is consistent with a previous finding of neutral optimal $\mathrm{pH}$ in many enzymes of LAB involved in carbohydrate and amino acid metabolism (11). It is interesting that in L. acidophilus JCM $1132^{\mathrm{T}}$, substantial glycolysis activity in the absence of nigericin was still observed at pH 7.5 or even at pH 8.5 (Fig. 3B), while the pHmax was 6.7 (Table 1). These results suggest that in this strain anabolic reactions may be more sensitive to an alkaline environment than catabolic reactions (glycolysis reactions).

Reversed $\Delta \mathrm{pH}$ formation was observed at alkaline external $\mathrm{pH}$ in all of the tested lactobacilli, as well as in E. hirae JCM $8729^{\mathrm{T}}$, with a magnitude of around 0.5 (Fig. 2). The dissipation of this $\Delta \mathrm{pH}$ by the addition of nigericin lowered the $\mathrm{pHmax}$ of each tested strain by 0.5 (Fig. 3A). These consistent results clearly indicate the contribution of the reversed $\Delta \mathrm{pH}$ to alkali tolerance. The formation of the reversed $\Delta \mathrm{pH}$ under alkaline external $\mathrm{pH}$ has already been reported for E. hirae ATCC $9790^{\mathrm{T}}(14,18,19)$, and such ATP-dependent $\mathrm{K}^{+} / \mathrm{H}^{+}$antiporters (15-17) and/or physicochemical forces as the Donnan potential (19) have been suggested to contribute to these phenomena. In the case of L. acidophilus JCM $1132^{\mathrm{T}}$, L. plantarum
NRIC 0380, and L. paracasei subsp. tolerans NRIC 1940, glucose utilization was still observed under the alkaline $\mathrm{pH}$ range in which the reversed $\Delta \mathrm{pH}$ was observed (Fig. 2 and $3 \mathrm{~B}$ ), thereby ensuring an energy supply under these conditions. Therefore, the observed reversed $\Delta \mathrm{pH}$ formation in these $\mathrm{Lac}$ tobacillus strains (Fig. 2) may possibly be mediated not only by some physicochemical force but also by certain energy-dependent $\mathrm{Na}^{+}\left(\mathrm{K}^{+}\right) / \mathrm{H}^{+}$antiporter(s). The larger reversed $\Delta \mathrm{pH}$ observed in L. acidophilus JCM $1132^{\mathrm{T}}$ when $\mathrm{NaOH}$ was used for $\mathrm{pH}$ adjustment than that observed when $\mathrm{KOH}$ was used (Fig. 2) suggested the possible operation of an $\mathrm{Na}^{+} / \mathrm{H}^{+}$antiporter. In this bacterium, since the pHmax is 6.7 (Table 1), the observed reversed $\Delta \mathrm{pH}$ (Fig. 2) may not contribute to the growth but may contribute to the survival of this bacterium under alkaline stress conditions.

We did not detect the remarkable regulation of cytoplasmic $\mathrm{pH}$ in E. hirae JCM $8729^{\mathrm{T}}$ (ATCC $9790^{\mathrm{T}}$ ) in external alkaline $\mathrm{pH}$ (Fig. 2) by such transporters as the ATP-driven $\mathrm{K}^{+} / \mathrm{H}^{+}$ antiporter reported previously for this bacterium (14-17). Similar results have been reported by Mugikura et al. (22). These discrepancies may have been caused by differences in the methods used for intracellular-pH measurements. It has been argued that the amines used for the measurement of internal $\mathrm{pH}$ by Kakinuma (14) tend to overestimate $\Delta \mathrm{pH}$ because of the binding of amines to intracellular materials such as RNA as well as to the cell surface (22). In contrast, Mugikura et al. (22) used weak acid for internal $\mathrm{pH}$ measurements, and its binding to intracellular materials is negligible. We used a $\mathrm{pH}$-sensitive fluorescent probe, cFSE, which allows the direct monitoring of intracellular $\mathrm{pH}$.

We investigated the physiological response of many Lactobacillus strains to an alkaline environment. The most important factor determining the diversity of alkali tolerance in lactobacilli was the alkali tolerance of the strains' glycolysis reactions. In addition, the reversed $\Delta \mathrm{pH}$ formed in alkaline environments, probably because of the Donnan potential and/or certain energy-dependent $\mathrm{Na}^{+}\left(\mathrm{K}^{+}\right) / \mathrm{H}^{+}$antiporter(s), contributed to the alkali tolerance. Although a variety of mechanisms aid LAB survival in acidic environments, lactobacilli seem to have poor evolutionary preparation for growth in alkaline environments. It has been reported that prior exposure to weak environmental stresses dramatically increases the tolerance to the corresponding stress in various LAB strains (3, 4, 28). Potential alkaline stress adaptation, as has been reported for E. faecalis ATCC $19433^{\mathrm{T}}$ (9), needs to be checked in lactobacilli for a more-comprehensive understanding of the mechanism of alkali tolerance. We have illustrated previously unexplored aspects of lactobacilli in alkaline conditions. We believe that these findings will help to understand the basic biology of lactobacilli and expand the application range of this group of bacteria.

\section{ACKNOWLEDGMENTS}

NRIC cultures were kindly supplied by Sanae Okada, Culture Collection Center, Tokyo University of Agriculture, Tokyo, Japan. $L$. plantarum WCFS1 was kindly donated by Michiel Kleerebezem, Wageningen Center for Food Sciences, Wageningen, The Netherlands.

A portion of this work was conducted under the framework of the Research and Development Program for New Bio-industry Initiatives, sponsored by the Bio-oriented Technology Research Advancement 
Institution (BRAIN). Y.S. received a scholarship from Kagome, Nagoya, Japan.

\section{REFERENCES}

1. Amachi, S., K. Ishikawa, S. Toyoda, Y. Kagawa, A. Yokota, and F. Tomita 1998. Characterization of a mutant of Lactococcus lactis with reduced membrane-bound ATPase activity under acidic conditions. Biosci. Biotechnol. Biochem. 62:1574-1580.

2. Breeuwer, P., J.-L. Drocourt, F. M. Rombouts, and T. Abee. 1996. A novel method for continuous determination of the intracellular $\mathrm{pH}$ in bacteria with the internally conjugated fluorescent probe 5 (and 6-)-carboxyfluorescein succinimidyl ester. Appl. Environ. Microbiol. 62:178-183.

3. Champomier-Vergès, M.-C., E. Maguin, M.-Y. Mistou, P. Anglade, and J.-F. Chich. 2002. Lactic acid bacteria and proteomics: current knowledge and perspectives. J. Chromatogr. B 771:329-342.

4. De Angelis, M., and M. Gobbetti. 2004. Environmental stress responses in Lactobacillus: a review. Proteomics 4:106-122.

5. Dellaglio, F., and G. E. Felis. 2005. Taxonomy of lactobacilli and bifidobacteria, p. 25-49. In G. W. Tannock (ed.), Probiotics and prebiotics: scientific aspects. Caister Academic Press, Norfolk, United Kingdom.

6. De Man, J. C., M. Rogosa, and M. E. Sharpe. 1960. A medium for the cultivation of lactobacilli. J. Appl. Bacteriol. 23:130-135.

7. Evans, D. F., G. Pye, R. Bramley, A. G. Clark, T. J. Dyson, and J. D. Hardcastle. 1988. Measurement of gastrointestinal $\mathrm{pH}$ profiles in normal ambulant human subjects. Gut 29:1035-1041.

8. Farrow, J. A. E., and M. D. Collins. 1985. Enterococcus hirae, a new species that includes amino acid assay strain NCDO 1258 and strains causing growth depression in young chickens. Int. J. Syst. Bacteriol. 35:73-75.

9. Flahaut, S., A. Hartke, J.-C. Giard, and Y. Auffray. 1997. Alkaline stress response in Enterococcus faecalis: adaptation, cross-protection, and changes in protein synthesis. Appl. Environ. Microbiol. 63:812-814.

10. Harold, F. M., and J. R. Baarda. 1968. Effects of nigericin and monactin on cation permeability of Streptococcus faecalis and metabolic capacities of potassium-depleted cells. J. Bacteriol. 95:816-823.

11. Hutkins, R. W., and N. L. Nannen. 1993. pH homeostasis in lactic acid bacteria. J. Dairy Sci. 76:2354-2365.

12. Ishikawa, M., K. Nakajima, M. Yanagi, Y. Yamamoto, and K. Yamasato. 2003. Marinilactibacillus psychrotolerans gen. nov., sp. nov., a halophilic and alkaliphilic marine lactic acid bacterium isolated from marine organisms in temperate and subtropical areas of Japan. Int. J. Syst. Evol. Microbiol. 53:711-720.

13. Ishikawa, M., K. Nakajima, Y. Itamiya, S. Furukawa, Y. Yamamoto, and K. Yamasato. 2005. Halolactibacillus halophilus gen. nov., sp. nov. and Halolactibacillus miurensis sp. nov., halophilic and alkaliphilic marine lactic acic bacteria constituting a phylogenetic lineage in Bacillus rRNA group 1. Int. J. Syst. Evol. Microbiol. 55:2427-2439.
14. Kakinuma, Y. 1987. Lowering of cytoplasmic $\mathrm{pH}$ is essential for growth of Streptococcus faecalis at high $\mathrm{pH}$. J. Bacteriol. 169:4403-4405.

15. Kakinuma, Y., and K. Igarashi. 1988. Active potassium extrusion regulated by intracellular $\mathrm{pH}$ in Streptococcus faecalis. J. Biol. Chem. 263: $14166-14170$

16. Kakinuma, Y., and K. Igarashi. 1995. Potassium/proton antiport system of growing Enterococcus hirae at high pH. J. Bacteriol. 177:2227-2229.

17. Kakinuma, Y., and K. Igarashi. 1999. Isolation and properties of Enterococcus hirae mutants defective in the potassium/proton antiport system. J. Bacteriol. 181:4103-4105.

18. Kobayashi, H. 1985. A proton-translocating ATPase regulates $\mathrm{pH}$ of the bacterial cytoplasm. J. Biol. Chem. 260:72-76.

19. Kobayashi, H., N. Murakami, and T. Unemoto. 1982. Regulation of the cytoplasmic pH in Streptococcus faecalis. J. Biol. Chem. 257:13246-13252.

20. Krulwich, T. A., A. A. Guffanti, R. F. Bornstein, and J. Hoffstein. 1982. A sodium requirement for growth, solute transport, and $\mathrm{pH}$ homeostasis in Bacillus firmus RAB. J. Biol. Chem. 257:1885-1889.

21. McLaggan, D., M. J. Selwyn, and A. P. Dawson. 1984. Dependence on $\mathrm{Na}^{+}$ of control of cytoplasmic $\mathrm{pH}$ in a facultative alkalophile. FEBS Lett. 165: 254-258.

22. Mugikura, S., M. Nishikawa, K. Igarashi, and H. Kobayashi. 1990. Maintenance of a neutral cytoplasmic $\mathrm{pH}$ is not obligatory for growth of Escherichia coli and Streptococcus faecalis at an alkaline pH. J. Biochem. 108:86-91.

23. Nakagawa, A., and K. Kitahara. 1959. Taxonomic studies on the genus Pediococcus. J. Gen. Appl. Microbiol. 5:95-126.

24. Ntougias, S., and N. J. Russell. 2001. Alkalibacterium olivoapovliticus gen. nov., sp. nov., a new obligately alkaliphilic bacterium isolated from edibleolive wash-waters. Int. J. Syst. Evol. Microbiol. 51:1161-1170.

25. Sánchez, A.-H., L. Rejano, A. Montaño, and A. de Castro. 2001. Utilization at high $\mathrm{pH}$ of starter cultures of lactobacilli for Spanish-style green olive fermentation. Int. J. Food Microbiol. 67:115-122.

26. Sawatari, Y., H. Sugiyama, Y. Suzuki, A. Hanaoka, K. Saito, H. Yamauchi, S. Okada, and A. Yokota. 2005. Development of fermented instant Chinese noodle using Lactobacillus plantarum. Food Microbiol. 22:539-546.

27. Toffin, L., K. Zink, C. Kato, P. Pignet, A. Bidault, N. Bienvenu, J.-L. Birrien, and D. Prieur. 2005. Marinilactibacillus piezotolerans sp. nov., a novel marine lactic acid bacterium isolated from deep sub-seafloor sediment of the Nankai Trough. Int. J. Syst. Evol. Microbiol. 55:345-351.

28. van de Guchte, M., P. Serror, C. Chervaux, T. Smokvina, S. D. Ehrlich, and E. Maguin. 2002. Stress responses in lactic acid bacteria. Antonie Leeuwenhoek 82:187-216.

29. Yokota, A., S. Amachi, S. Ishii, and F. Tomita. 1995. Acid sensitivity of a mutant of Lactococcus lactis subsp. lactis $\mathrm{C} 2$ with reduced membrane-bound ATPase activity. Biosci. Biotechnol. Biochem. 59:2004-2007. 